\title{
La cooperación tecnológica entre bibliotecas digitales académicas de México
}

\author{
Georgina Araceli Torres Vargas *
}

Artículo recibido:

3 de abril de 2013.

Artículo aceptado:

7 de agosto de 2013.

\section{Resumen}

Se analiza si existe cooperación entre las bibliotecas digitales académicas mexicanas para desarrollar aplicaciones tecnológicas que ayuden en el mejoramiento de sus actividades. El estudio se basa en un modelo teórico de la biblioteca digital previamente armado, en donde la variable tecnológica sirve como punto de partida para conocer cómo se establece la cooperación entre las bibliotecas digitales académicas de México. Los resultados que se arrojan ayudan a conocer cómo se da la cooperación entre bibliotecas digitales académicas en el contexto mexicano.

Palabras clave: Biblioteca Digital Académica; Bibliotecas digitales mexicanas.

* Instituto de Investigaciones Bibliotecológicas y de la Información de la UNAM, México.gatv@unam.mx

INVESTIGACIÓN BIBLIOTECOLÓGICA, Vol. 28, Núm.62, enero/abril, 2014, México, ISSN: 0187-358X. pp. 67-79 


\section{Abstract}

Cooperation in the area of technology among Mexican digital academic libraries

Georgina-Araceli Torres-Vargas

In this paper researchers examine the actuality of cooperation between Mexican digital academic libraries in the area of developing technological applications to improve operational performance. The study is based on a previously developed theoretical digital library model, in which the technological variable serves as a starting point to learn how to enhance cooperation between academic digital libraries in Mexico. The results help shed light on the degree and nature of cooperation between academic digital libraries in Mexico.

Keywords: Academic Digital Library; Mexican digital libraries.

\section{INTRODUCCIÓN}

$\mathrm{C}^{1}$ presente trabajo muestra datos sobre la cooperación para el desarrollo Dde aplicaciones tecnológicas.

Esta investigación parte de dos aspectos metodológicos: el teórico y el empírico. La vertiente teórica se basa en un modelo de biblioteca digital (BD) resultado de una investigación inmediata anterior, en donde se establecieron los elementos que integran a una biblioteca digital; como elemento empírico se utilizó la metodología de redes sociales para obtener un mapeo de las relaciones de cooperación que se establecen entre el personal de bibliotecas digitales académicas mexicanas para desarrollar aplicaciones útiles en la biblioteca.

El desarrollo de teCNOlogías PARA LA Biblioteca digital

Para comenzar es preciso partir de la definición que he propuresto para la biblioteca digital (BD): 
La biblioteca digital es una biblioteca que se sustenta en un sistema de información documental en red, que ofrece a sus usuarios contenidos y servicios digitales. ${ }^{1}$

La BD se integra por las siguientes variables:

1. Tecnologías de la información y la comunicación necesarias para el acceso a colecciones de la BD.

2. Contenidos digitales organizados. Se encuentran distribuidos en diversos niveles de red, con distintos niveles de accesibilidad. Pueden ser al mismo tiempo locales y compartidos.

3. Servicios digitales. Se prestan y administran servicios en cooperación con otras bibliotecas

En este caso nos concentraremos en el elemento tecnológico. Si bien la tecnología no es el centro del desarrollo de una biblioteca digital, es innegable que cuenta con una importancia considerable. La conformación de la biblioteca digital depende en gran medida de los avances de las tecnologías de la información y de la comunicación.

Sin embargo, no es deseable la integración de tecnologías sólo por el hecho de ser novedosas, sino que es importante analizar los fines que se persiguen en el desarrollo de colecciones y en el ofrecimiento de servicios que, como se mencionó, son las otras variables que dan estructura a la biblioteca digital.

Por otra parte es bien conocido que las TIC que se generan aparecen en contextos muy diversos y que la mayor parte de las ocasiones no están destinadas a la biblioteca. Es por eso que cada vez es más necesario lograr desarrollos tecnológicos enfocados a la biblioteca digital, en donde se puedan manifestar servicios nuevos y mejores usos de la información digital así como la agilización de procesos, entre otras cuestiones.

El desarrollo de tecnologías dirigidas a la biblioteca digital ayudaría a buscar una armonía con las otras variables o componentes de la biblioteca, pero sobre todo se dirigirían al apoyo de las necesidades específicas de la población usuaria.

En el caso de la biblioteca digital académica es importante conocer cuáles son los usos de la información que demanda el profesor, el investigador o el estudiante, para encaminar así los esfuerzos hacia el diseño y la implementación de tecnologías apropiadas. Pero en este caso es necesario no perder de

1 Esta definición ya ha sido referida y detallada en otros trabajos, véase Georgina Araceli Torres Vargas, Un modelo integral de biblioteca digital, México, CUIB, 2008. 
vista un enfoque social, en donde no sólo se atiendan las herramientas sino también los contenidos y los servicios; lograr esto es uno de los objetivos más importantes de toda biblioteca, sea ésta o no digital. El servicio descansa en las colecciones, que a su vez están determinadas por las necesidades de información de la comunidad.

El desarrollo de tecnologías aplicadas a la BD es un tema que no parece haber sido analizado con detenimiento. La mayor parte de la literatura detalla las tecnologías que se han adoptado en el seno de la biblioteca y si bien en ocasiones se habla de innovación, ésta se entiende de forma errónea pues se piensa que innovar es utilizar tecnologías de punta en la biblioteca.

Pero la innovación debe entenderse como un proceso que consta de etapas (científicas, técnicas, comerciales y financieras necesarias para el desarrollo y comercialización de productos nuevos o con mejoras), e implica la utilización de nuevos o mejores procesos y equipos o la introducción de un nuevo servicio. ${ }^{2} \mathrm{El}$ proceso de innovación concluye cuando el producto es introducido en el mercado, o cuando es utilizado. ${ }^{3}$

En el caso de la biblioteca la innovación puede ser mucho más visible en los servicios que en cualquier otra área, pero también es necesaria en los procesos internos, además de otros aspectos del quehacer bibliotecario. En este sentido es útil la categorización que ofrece $\mathrm{Merlo}^{4}$ para identificar las posibles aplicaciones tecnológicas en la biblioteca digital y observar las áreas en las que es posible generar innovaciones tecnológicas:

- Desarrollo de colecciones (en la BD se puede referir a la creación de contenidos digitales).

- Organización de colecciones.

- Difusión de la colección.

- Actividad profesional.

A esta lista se agregarían los servicios digitales. En cada uno de estos aspectos es necesario llevar a cabo un trabajo que va desde la identificación de necesidades hasta el desarrollo e implementación de innovaciones en la biblioteca. Esta labor se puede realizar en forma cooperativa, sobre todo en el entendido de que en el contexto de la biblioteca digital se está trabajando en redes de bibliotecas digitales.

Manuel Ruiz González, La innovación tecnológica y su gestión, Barcelona, MARCOMBO, 1989, p. 14. 3 Ibid., p. 20.

4 José Antonio Merlo Vega, "50 aplicaciones bibliotecarias en Internet", en Educación y Biblioteca, núm. 106, noviembre, 1999, pp. 38-47. 
Gran parte del trabajo en cooperación ${ }^{5}$ entre bibliotecas digitales se da a través de $\operatorname{consorcios}^{6}$ que dan relevancia a la adquisición de contenidos electrónicos y a tener acceso a paquetes de software. ${ }^{7}$

Los consorcios se caracterizan por:

- Crearse mediante un acuerdo formal entre las instituciones implicadas, con compromisos firmados.

- Diseñar un plan conjunto.

- Contar con sistemas de organización y control que permiten asegurar el cumplimiento de sus objetivos.

Se ha visto que en los consorcios no se atiende el trabajo en conjunto cuando se busca el desarrollo de aplicaciones tecnológicas. En relación con esto, Anglada ${ }^{8}$ menciona que en España quizá el principal punto débil en los consorcios de bibliotecas sea la falta de apoyo informático para desarrollar proyectos de forma innovadora. Subraya la necesidad de establecer colaboraciones efectivas dentro de la universidad que permitan avanzar al ritmo de los avances tecnológicos e innovar en los servicios.

En relación con ellos se puede mencionar un estudio ${ }^{9}$ en donde se detalló cómo la biblioteca digital no cuenta con servicios propios, sino que ha desarrollado servicios dirigidos a potencializar aquellos que ya se ofrecían en la biblioteca convencional. Es decir, todavía hoy, a más de una década de haber aparecido la idea de biblioteca digital, no se ha dado el salto que se requiere para conformar un universo de lo digital separado de lo impreso, en este caso en el ámbito de los servicios. Además de esta área es necesario desarrollar tecnologías que apoyen los aspectos bibliotecarios que señala Merlo.

En el caso de México el consorcio en bibliotecas académicas no ha obtenido los beneficios esperados, sobre todo por la falta de compromiso para

5 Anglada señala que la cooperación es el establecimiento de fuertes lazos con bibliotecas que tienen elementos comunes (sea por la proximidad territorial, la tipología o la especialidad) En la cooperación no sólo se comparten ideas sino también, recursos. Cfr. Lluís M. Anglada i de Ferrer, "Colaboraciones y alianzas: la inteligencia social aplicada a las bibliotecas universitarias", en Anales de Documentación, núm. 9, pp. 7-15.

6 El consorcio es una sociedad formada por un grupo de bibliotecas que se constituye para desarrollar y compartir los recursos de todos los miembros y para mejorar los servicios bibliotecarios y los recursos de los que disponen. Cfr. Heartsill Young, Glosario ALA de bibliotecología y ciencias de la información, Madrid, Díaz de Santos, 1998.

7 Miquel Térmens Graells, "Los consorcios, una nueva etapa de la cooperación bibliotecaria", en Elprofesional de la información, vol. 14, núm. 3, 2005, pp. 164-173.

8 Anglada, op. cit., p. 14

9 Georgina Araceli Torres Vargas, Hacia un modelo de servicios en la biblioteca digital, en Investigación Bibliotecológica, vol. 37, núm. 35, julio-diciembre, 2003, pp. 32-44 
fortalecer el propio consorcio, ${ }^{10}$ lo cual se traduce en una cooperación desequilibrada que no toma en cuenta que la mayor o menor cooperación no debe darse de acuerdo con la cantidad de presupuesto que aporte cada biblioteca, sino que la equidad en la participación sólo puede darse a través del aporte de muchos otros elementos.

Uriarte señala "que la meta de los consorcios bibliotecarios [...] va más allá de la adquisición compartida, la donación, el préstamo interbibliotecario, o la recuperación de documentos [...]"11 y acota que el consorcio deberá enfrentar los nuevos cambios e innovaciones que llegan del mundo de la información electrónica. En concordancia con esta aseveración, se observa que los consorcios de bibliotecas académicas son un factor determinante para la creación y el uso de aplicaciones tecnológicas que impregnen las diferentes áreas de la biblioteca, pero que en primera instancia enriquezcan el área de servicios y que éstos ofrezcan ventajas novedosas a los usuarios que pertenecen a la red.

La colaboración entre bibliotecas digitales con fines similares constituiría de manera natural un consorcio, pero no es algo que deba hacerse por obligación. Por lo general las bibliotecas digitales trabajan cooperativamente en redes y no en consorcios.

Anteriormente se señaló que la BD es un sistema de información documental en red y que como tal se estructura en nodos. Las redes son estructuras abiertas capaces de expandirse sin límites; no tienen un centro y pueden crecer añadiendo más nodos. ${ }^{12}$ Bajo esta consideración se mencionan en seguida dos redes de bibliotecas académicas mexicanas y se analizan en cuanto a la cooperación que establecen en el desarrollo de tecnologías.

\section{LA COOPERACIÓN TECNOLÓGICA ENTRE BIBLIOTECAS DIGITALES aCADÉmicas en MéXico a través de un estudio de REDES SOCIALES}

Este estudio se centra en la biblioteca digital académica debido a que en la actualidad es el tipo de biblioteca que más impulso recibe a nivel mundial gracias a las ventajas que muestra para su comunidad. Las redes de bibliotecas digitales académicas mexicanas que más se acercan al modelo de biblioteca digital planteado teóricamente son dos:

10 Lucía Uriarte Franco, Consorcios en bibliotecas académicas, México, 2007, Tesis, UNAM, Facultad de Filosofía y Letras, Posgrado en Bibliotecología y Estudios de la Información, p. 135. 11 Ibid., p. 136.

12 Anglada, op. cit., pp. 10-11. 
1. La Red Abierta de Bibliotecas Digitales (RABID) Su objetivo principal es

[...] contribuir a la consolidación del desarrollo de bibliotecas digitales en México a través de una red abierta por medio de la cual puedan compartirse colecciones y servicios disponibles en diferentes instituciones mientras se facilita la integración de nuevas instituciones, servicios y usuarios. ${ }^{13}$

En esta red participan 15 universidades, entre ellas la Benemérita Universidad Autónoma de Puebla (BUAP), el Instituto Tecnológico y de Estudios Superiores de Monterrey (ITESM Campus Monterrey) y la Universidad de Guadalajara (UDG), ${ }^{14}$ y se ha atendido el aspecto tecnológico en lo siguiente:

a. Desarrollo y creación de contenidos digitales. Cuenta con contenidos digitales como son algunas colecciones de tesis, acervos antiguos digitalizados, revistas electrónicas de una institución participante y el acceso a una red de revistas electrónicas.

b. Difusión de la colección. Es un sistema de información documental en red, que busca dar acceso a repositorios institucionales.

2. La Red de Bibliotecas Digitales y Consorcio Bibliotecario ECOES La misión del Espacio Común de Educación Superior (ECOES) es fortalecer los espacios educativos nacional, latinoamericano y del Caribe que permitan la conjunción de esfuerzos institucionales para transformar la educación superior e innovar los modelos de formación académica en el contexto de la sociedad del conocimiento.

Actualmente ECOES está integrado por 30 Instituciones de Educación Superior (IES) que agrupan a la mitad de la población estudiantil de educación superior de México. ${ }^{15}$ El objetivo de la Red de Bibliotecas Digitales y Consorcios Bibliotecarios ECOES es consolidar una red de bibliotecas digitales mediante la cooperación e integración de las instituciones participantes, en apoyo a los programas ECOES. ${ }^{16}$

Tecnológicamente, esta red ha puesto atención en el desarrollo y la creación de contenidos digitales, los cuales promueve en cada una de las instituciones participantes.

13 Red Abierta de Bibliotecas Digitales RABID, disponible en: http://ict.udlap.mx/rabid/index_ es.html\#documentos

14 Para más detalles sobre las dos redes véase Georgina Araceli Torres Vargas, "La biblioteca digital académica en México a través de dos casos", en Memoria del Quinto Seminario Hispano Mexicano de Investigación en Bibliotecología y Documentación, 9, 10 y 11 de abril del 2008, Filiberto Felipe Martínez Arellano (comp.) México, UNAM, CUIB, 2009, pp. 219-231, disponible en: http://132.248.242.3/ publica/archivos/libros/5o_seminario_hispanomexicano.pdf

15 Información disponible en: http://www.ecoes.unam.mx/

16 Información disponible en: http://www.ecoes.unam.mx/red_biblio_pvp.html 
En general, se busca facilitar el intercambio de conocimientos en tecnologías de la información y la comunicación.

Como ya se dijo, uno de los rasgos básicos de una red de bibliotecas digitales es la cooperación, la cual debe darse en diferentes niveles:

- Compartiendo contenidos digitales entre sus comunidades (contenidos distribuidos).

- Ofreciendo servicios de información compartidos.

- Generando aplicaciones tecnológicas en grupo y utilizándolas entre los participantes de la red, sea para la organización y recuperación de colecciones, para el ofrecimiento de servicios o para el seguimiento de procesos y la comunicación entre bibliotecarios de las instituciones que conforman la red.

Luego de analizar los diferentes documentos de trabajo de estas dos redes, se elaboraron las redes sociales ${ }^{17}$ que permiten mapear las relaciones de cooperación tecnológica que se han establecido. Hay que mencionar que los resultados se graficaron con ayuda de Pajek, software que permite realizar análisis de redes sociales para identificar el tipo de relaciones que se han establecido entre las bibliotecas de cada red, y de esta forma conocer hacia dónde se dirige su desarrollo.

De esta forma se obtuvieron los siguientes sociogramas:

1. Cooperación de instituciones con consorcios

2. Redes de cooperación y aplicaciones tecnológicas

3. Aplicaciones tecnológicas e instituciones

Se observa que las instituciones que forman parte de RABID también colaboran en algún consorcio (en el sociograma los consorcios se grafican en el nodo "otros"), y que nueve de los participantes de RABID también son integrantes de la red ECOES (UAEH, UNAM, BUAP, UASLP, UAA, IPN, UDEG, UAEM, UV). El resto de los participantes colaboran exclusivamente con ECOES.

El sociograma de la Figura 3 muestra que ECOES cuenta con tres desarrollos o implementaciones para las bibliotecas. Por su parte, RABID cuenta con cerca de 24 desarrollos o implementaciones para los participantes.

17 Las redes sociales ayudan a mostrar cualquier hecho que involucre una intencionalidad colectiva. Estos hechos no pueden verse a simple vista, es necesario acercarse a ellos a través del conocimiento de las prácticas que se llevan a cabo al interior de la institución, y un medio para lograrlo son las redes sociales. Para algunos autores el método de redes significa el mapeo que hace comprensible lo "real". Este mapeo se grafica a través de sociogramas. 
LA COOPERACIÓN TECNOLÓGICA ENTRE BIBLIOTECAS DIGITALES...

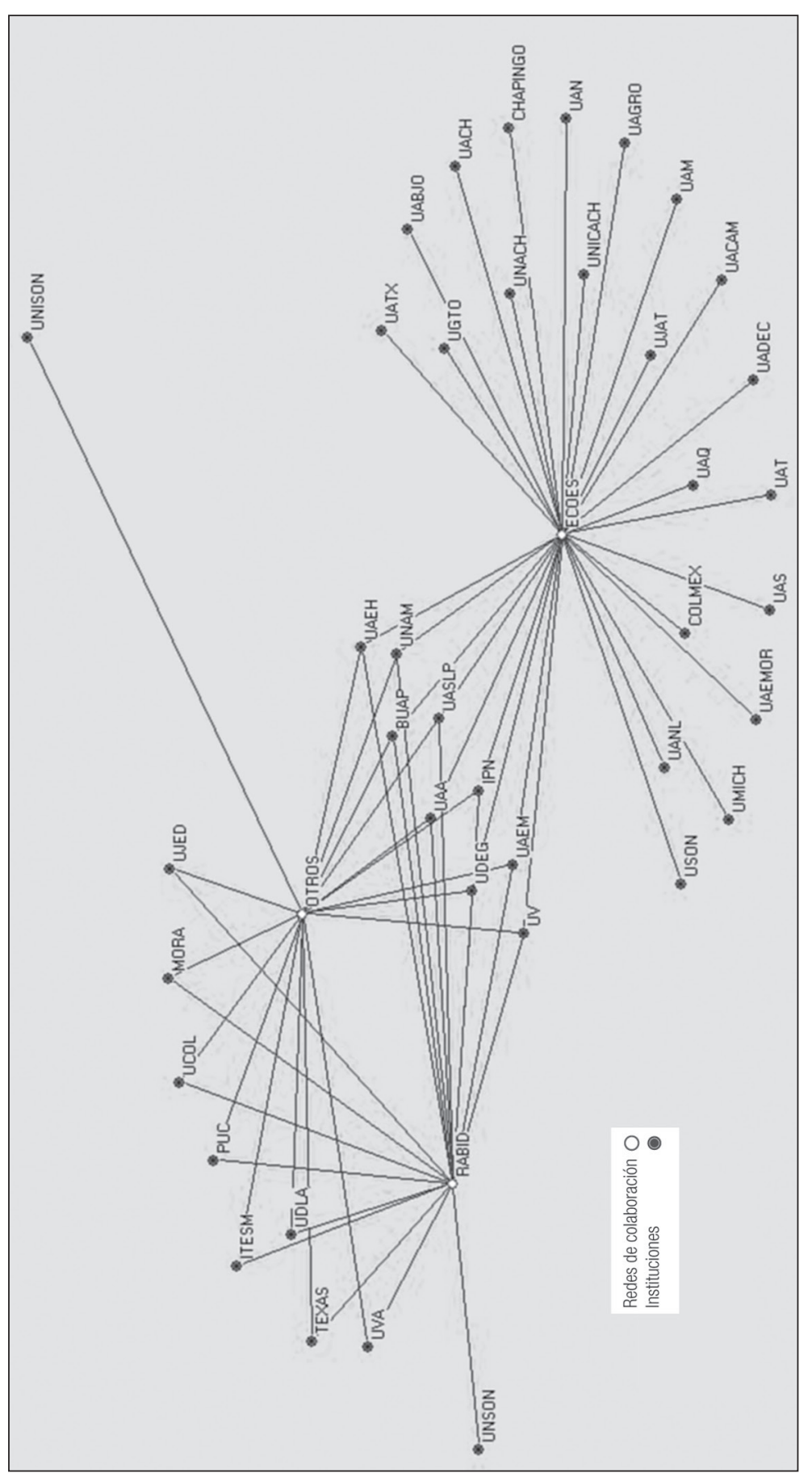

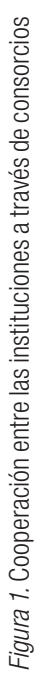




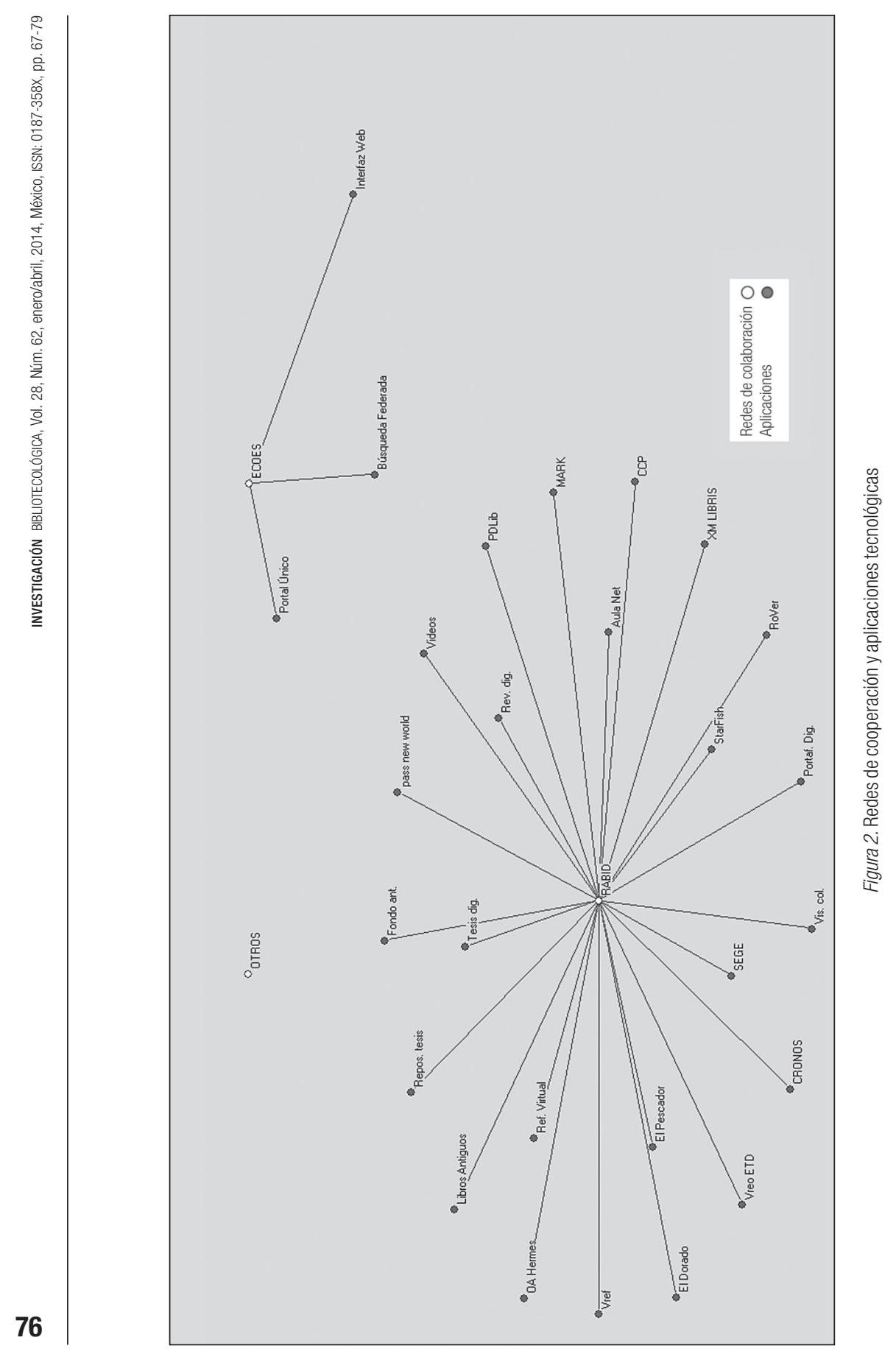


LA COOPERACIÓN TECNOLÓGICA ENTRE BIBLIOTECAS DIGITALES...
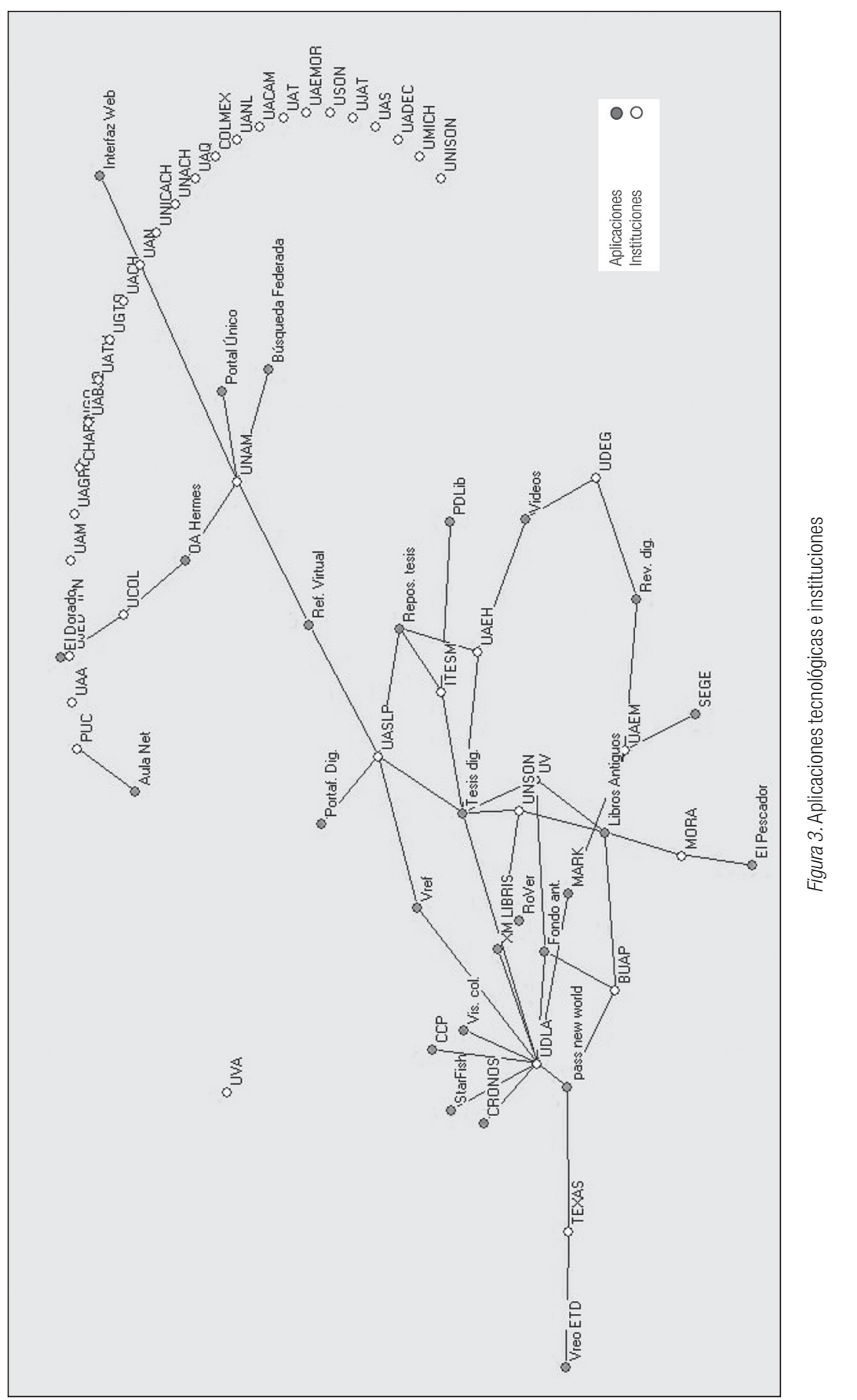
Aquí se revela un desequilibrio en cuanto a las aportaciones de los integrantes de las dos redes, ya que sólo unas cuantas instituciones ofrecen desarrollos. También se percibe el liderazgo de muy pocas instituciones en ambas redes.

Muchos de los casos reflejan la adhesión a las redes para beneficio individual, ya que diversas instituciones aparecen como integrantes de estos grupos pero en realidad no hacen aportes, fuera de que algunos de sus integrantes ofrecen cursos relacionados con lo que se trabaja en las redes.

En cuanto a las implementaciones cabe resaltar que el diseño de metabuscadores es frecuente, aunque por el estado actual en el que éstos se encuentran no forman parte de una biblioteca digital, ya que si bien estos sistemas ayudan a identificar la ubicación de los documentos dentro de los acervos de todas las bibliotecas participantes no implica que dichos documentos sean digitales.

Lo mismo ocurre cuando se relacionan catálogos en línea de algunas bibliotecas a través de la página web de la red. Esta relación no representa un desarrollo de la biblioteca digital, sino un medio que ayuda en el ofrecimiento de servicios de la biblioteca convencional o tradicional, sólo que a través de medios electrónicos.

Por tales razones es necesario resaltar que un alto porcentaje de implementaciones no son propias de una biblioteca digital, sino que potencializan los procesos y servicios de la biblioteca convencional.

Por otra parte, la pertenencia a consorcios por parte de algunas universidades (consorcios como Grupo Amigos y el CONPAB-IES, Consejo Nacional para Asuntos Bibliotecarios de Instituciones de Educación Superior, A. C.) refleja la gran necesidad que se tiene de comprar licencias mediante estas agrupaciones y, por consiguiente, la carencia que hay en el desarrollo de contenidos digitales propios, lo que impide la cooperación en este rubro y por ende la dependencia de los proveedores de contenidos en la constitución de bibliotecas digitales. Fuera de las tesis digitales y de los materiales antiguos, es muy escaso el contenido digital del que se dispone en las instituciones de educación superior en México.

\section{Conclusiones}

De acuerdo con lo hasta aquí mencionado se observa que:

a. Diversas aplicaciones que han sido diseñadas por algunas instituciones se han adecuado para su implementación entre los participantes de las redes. 
b. Se notó también la relación de algunas universidades con otros grupos de bibliotecas reunidas en consorcios, tales como el Grupo Amigos y CONPAB-IES. Estas relaciones se muestran en la gráfica, mediante el nodo "otros".

c. La adhesión a las redes de bibliotecas digitales no garantiza una aportación de todos los participantes que redunde en beneficios del grupo. Muchas de las instituciones se convierten en simples receptoras de desarrollos hechos por otras universidades. La adopción de desarrollos tecnológicos hechos por otros crea una falta de autonomía, pero sobre todo tiende a apegarse más a las necesidades de quienes crean estos desarrollos. Es necesario que todas las instituciones participen con propuestas propias y que integren sus necesidades a la colectividad.

d. Las universidades han tendido hacia una integración en más de una red de bibliotecas, ya que en cada una de ellas obtienen beneficios particulares que de cierta forma pueden complementarlas. Esto en cierta forma refleja la individualidad con la que se está trabajando en cuanto a la construcción de bibliotecas digitales académicas.

e. La implementación de desarrollos desde diferentes orígenes y propósitos evita que se cuente con un proyecto cohesivo y a largo plazo de bibliotecas digitales.

f. Hace falta la reagrupación en una gran red que, mediante un acuerdo formal, establezca derechos y obligaciones de cooperación equilibrada; que se establezca un plan de desarrollo basado en una idea más cercana a lo que debe ser una biblioteca digital, y que exista la determinación de grupos líderes en cada uno de las diversas áreas de trabajo.

Si no se revisa y corrige la forma en que se está trabajando, el futuro de la biblioteca digital académica en México será poco esperanzador.

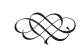

